\title{
Reconstruction of Spatially Resolved Pharmacokinetic Rate Images of Fluorescence Agents in FDOT
}

\author{
Burak Alacam, Birsen Yazici \\ Ahmed Serdaroglu \\ Dept. of Electrical, Computer, and \\ Systems Engineering \\ Rensselaer Polytechnic Institute \\ Troy, New York, 19102
}

\author{
Xavier Intes \\ ART Inc. \\ Saint-Laurent, Quebec \\ H4S 2A4, Canada
}

\author{
Britton Chance, Shoko Nioka \\ Department of Biochemistry and Biophysics, \\ University of Pennsylvania, \\ Philadelphia, PA, 19104
}

\begin{abstract}
In this work, we propose a method to reconstruct spatially resolved pharmacokinetic rate images of fluorescence agents directly from the boundary photon flux measurements. We use a compartmental modeling scheme to model the pharmacokinetics of fluorescence agents. We coupled this model with the fluorescence diffuse optical tomography (FDOT) forward model to form a state space model which is then iteratively solved by extended Kalman filtering (EKF) algorithm. As an example, we used a two-compartment model for indocyanine green (ICG) pharmacokinetics. To validate the proposed method, we tested our approach using a simulation study. Reconstructed pharmacokinetic rate images with correct localization of heterogeneities and high reconstruction accuracy show that the proposed algorithm can be used for tumor detection, tumor diagnosis, drug delivery and feasibility studies.
\end{abstract}

\section{INTRODUCTION}

Development of fluorescence agents that emit light upon excitation lead to a new imaging technique, called fluorescence diffuse optical tomography (FDOT), whose theory is a straightforward extension of diffuse optical tomography. In FDOT, near infrared (NIR) excitable exogenous fluorescence agents, which are externally injected into the tissue are investigated in terms of their optical parameters [1]. These agents enhance the sensitivity of detection hence increase the optical contrast. FDOT has the ability to characterize functional parameters of tissue such as scattering and absorption, which can be used to derive pharmacokinetics of fluorescence agents.

The analysis of pharmacokinetics of fluorescence agents is a potential means for tumor detection, diagnosis, drug delivery and feasibility studies [2]. One approach to pharmacokinetic analysis is the compartmental modeling [6]. In this method, a region of interest consists of a number of compartments, generally representing a volume of similar tissues into which the fluorescence agent is distributed. The concentration changes in a specific compartment are modeled as a result of the exchange of fluorescence agents between connected compartments. These changes are modeled by a collection of coupled ordinary differential equations (ODE); each equation describing the time change dictated by the biological laws that govern the concentration exchanges between the interacting compartments. Coefficients of the ODE's are the pharmacokinetic rates that represent rates of exchange between different compartments.

A number of research groups reported compartmental modeling of fluorescence agents for tumor diagnosis in animal and human subjects [3]-[5]. In these studies, bulk values that represent pharmacokinetic rates either over the entire imaging domain or a large volume is used for tumor detection/diagnosis. In such cases, spatially resolved representation of pharmacokinetic rates may increase specificity and sensitivity compared to average bulk rates. Moreover, there are studies in literature showing that the spatially varying pharmacokinetics rates are quantitatively different inside and outside the tumor region [7]-[9].

In this paper, we proposed a compartmental modeling scheme for fluorescence agent pharmacokinetics, and reconstructed spatially resolved pharmacokinetic rate images directly from the boundary photon flux measurements. We also reconstructed the spatially resolved fluorescence agent concentration images inside different compartments. In our algorithm, we used a finite element method (FEM) [10] as a forward solver and an extended Kalman filter (EKF) algorithm [3] as an inverse solver. We tested our approach using a simulated data set based on a two-compartment model for ICG pharmacokinetics. For the simulation studies, we generated a set of time series data from a domain with tissue-like characteristics using FEM algorithm to solve the coupled diffusion equations with Robin type boundary conditions. Reconstructed pharmacokinetic images with correct localization of heterogeneities and high reconstruction accuracy show that the proposed algorithm can be useful for tumor diagnosis, drug delivery and feasibility studies.

The rest of the paper is organized as follows: In Section II, we present pharmacokinetic analysis and compartmental modeling for fluorescence agents. In Section III, we present FDOT forward problem. Section IV describes the inverse problem and the EKF algorithm for direct reconstruction of pharmacokinetic parameters. In Section V, we present the simulation results. Section VI summarizes our results and conclusion. 


\section{Pharmacokinetic Modeling And COMPARTMENTAL MOdEl ANALYSiS}

In general, the continuous time state-space representation for an $n$-compartment model is given by

$$
\begin{aligned}
\dot{\mathbf{C}}(t) & =\mathbf{K}\left(\boldsymbol{\alpha}_{n}\right) \mathbf{C}(t) \\
m(t) & =\mathbf{V}\left(\boldsymbol{\alpha}_{n}\right) \mathbf{C}(t)
\end{aligned}
$$

where $\mathbf{C}(t)$ denotes the concentration vector whose elements are the concentrations of the fluorescence agents in different compartments, and $\dot{\mathbf{C}}(t)$ denotes its time derivative. $m(t)$ is the bulk fluorescence concentration, $n$ is the number of compartments, $\boldsymbol{\alpha}_{n}$ is the parameter vector whose elements are the pharmacokinetic rate constants and volume fractions. $\mathbf{K}\left(\boldsymbol{\alpha}_{n}\right)$ is the system matrix with entries being the pharmacokinetic rates, and $\mathbf{V}\left(\boldsymbol{\alpha}_{n}\right)$ is the vector containing the volume fractions [6].

Although the formulation for the direct reconstruction of pharmacokinetic rate images can be applicable for ncompartment models, here the two-compartment model for ICG pharmacokinetics will be our running example [3], [4]. Based on a two-compartment model for ICG kinetics, as shown in Figure 1, the ICG transition between two compartments can be modeled by two coupled differential equations:

$$
\begin{gathered}
\frac{d C_{e}(t)}{d t}=-k_{\text {out }} C_{e}(t)+k_{i n} C_{p}(t), \\
\frac{d C_{p}(t)}{d t}=-\left(k_{\text {in }}+k_{\text {elm }}\right) C_{p}(t)+k_{\text {out }} C_{e}(t) .
\end{gathered}
$$

Here, $\mathbf{C}(t)$ in (1) and (2) includes, $C_{p}(t)$ and $C_{e}(t)$, representing the ICG concentrations in the plasma and extracellular extravascular space (EES), respectively. The parameter vector, $\boldsymbol{\alpha}_{2}$, includes, $k_{\text {in }}, k_{\text {out }}, k_{e l m}, v_{p}$, and $v_{e}$;

$$
\boldsymbol{\alpha}_{2}=\left[\begin{array}{lllll}
k_{\text {in }} & k_{\text {out }} & k_{\text {elm }} & v_{p} & v_{e}
\end{array}\right]^{T},
$$

where $k_{\text {in }}$ and $k_{\text {out }}$ are the pharmacokinetic rates that govern the leakage into and the drainage out of the EES, $k_{e l m}$ describes the ICG elimination from the body through kidneys and livers, and $v_{p}$ and $v_{e}$ are the plasma and EES volume fractions, respectively.

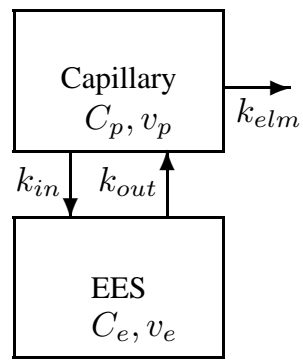

Fig. 1. Block diagram of the two-compartment model for ICG pharmacokinetics.

Here, to obtain 2-D images of pharmacokinetic rates and fluorescence agent concentrations in different compartments, we extend the compartmental model equations (1) and (2) to spatially resolved model as follows:

$$
\begin{aligned}
{\left[\begin{array}{c}
\dot{\mathbf{C}}\left(r_{1}, t\right) \\
\vdots \\
\dot{\mathbf{C}}\left(r_{N}, t\right)
\end{array}\right]=} & {\left[\begin{array}{ccc}
\mathbf{K}\left(\boldsymbol{\alpha}_{n}\left(r_{1}\right)\right) & 0 & 0 \\
0 & \ddots & 0 \\
0 & 0 & \mathbf{K}\left(\boldsymbol{\alpha}_{n}\left(r_{N}\right)\right)
\end{array}\right]\left[\begin{array}{c}
\mathbf{C}\left(r_{1}, t\right) \\
\vdots \\
\mathbf{C}\left(r_{N}, t\right)
\end{array}\right] } \\
& +\quad \boldsymbol{\omega}(\mathbf{r}, t)
\end{aligned}
$$

where $\boldsymbol{\omega}(\mathbf{r}, t)$ is uncorrelated zero mean Gaussian processes with covariance matrix $\mathbf{Q}$, representing the small deviations resulting from model mismatch. $\mathbf{r}=\left[\begin{array}{lll}r_{1} & r_{2} \ldots r_{N}\end{array}\right]$, and $r_{j}$ is the location of the $j^{t h}$ voxel for $j=1,2,3 \ldots N, N$ being the number of total voxels in the discritized domain.

$$
\begin{aligned}
{\left[\begin{array}{c}
m\left(r_{1}, t\right) \\
\vdots \\
m\left(r_{N}, t\right)
\end{array}\right]=} & {\left[\begin{array}{ccc}
\mathbf{V}\left(\boldsymbol{\alpha}_{n}\left(r_{1}\right)\right) & 0 & 0 \\
0 & \ddots & 0 \\
0 & 0 & \mathbf{V}\left(\boldsymbol{\alpha}_{n}\left(r_{N}\right)\right)
\end{array}\right]\left[\begin{array}{c}
\mathbf{C}\left(r_{1}, t\right) \\
\vdots \\
\mathbf{C}\left(r_{N}, t\right)
\end{array}\right] } \\
& +\boldsymbol{\eta}(\mathbf{r}, t)
\end{aligned}
$$

where $\boldsymbol{\eta}(\mathbf{r}, t)$ is a zero mean Gaussian process with covariance matrix $\mathbf{R}$ presenting the noise in the measurements.

The implicit form of (6) and (7) are given by:

$$
\begin{aligned}
& \dot{\mathbf{C}}(\mathbf{r}, t)=\mathbf{K}\left(\boldsymbol{\alpha}_{n}(\mathbf{r})\right) \mathbf{C}(\mathbf{r}, t)+\boldsymbol{\omega}(\mathbf{r}, t) \\
& \mathbf{m}(\mathbf{r}, t)=\mathbf{V}\left(\boldsymbol{\alpha}_{n}(\mathbf{r})\right) \mathbf{C}(\mathbf{r}, t)+\boldsymbol{\eta}(\mathbf{r}, t)
\end{aligned}
$$

The quantity we wish to reconstruct is the spatially varying pharmacokinetic rate parameters instead of the absorption coefficient of the florescence agents as commonly the case in FDOT [1]. To do this, we first need to develop a mapping which relates the boundary flux measurements to the bulk florescence agent concentrations in tissue. This mapping can be obtained by using FDOT forward model which will be explained in the next section.

\section{Fluorescence Diffuse Optical Tomography}

We used a coupled system of diffusion equations to model fluorescence light propagation in tissue [1]. Based on the coupled diffusion equations, the forward model for FDOT can be expressed as:

$$
\boldsymbol{\Psi}(\mathbf{r}, t)=f\left(\boldsymbol{\mu}_{a f}(\mathbf{r}, t)\right),
$$

where $\boldsymbol{\mu}_{a f}(\mathbf{r}, t)=\left[\mu_{a f}\left(r_{1}, t\right), \cdots, \mu_{a f}\left(r_{N}, t\right)\right]$ is the time dependent absorption coefficient of the fluorescence agent, $\boldsymbol{\Psi}(\mathbf{r}, t)=\left[\Psi\left(r_{1}, t\right), \cdots, \Psi\left(r_{N}, t\right)\right]$ is the time-dependent boundary flux measurements, and $f$ is a nonlinear function defined by the coupled diffusion equation. Under the assumption that the exogenous optical properties has no effect on endogenous optical properties, (10) can be linearized to obtain

$$
\boldsymbol{\Psi}(\mathbf{r}, t)=\mathbf{W} \boldsymbol{\mu}_{a f}(\mathbf{r}, t),
$$

where $\mathbf{W}$ is the weight matrix which maps the absorption coefficients to the boundary measurements obtained using the FEM algorithm [10].

The absorption coefficient of the fluorescence agents is related to the bulk fluorescence agent concentration as follows:

$$
\boldsymbol{\mu}_{a f}(\mathbf{r}, t)=\epsilon \mathbf{m}(\mathbf{r}, t)
$$


where $\epsilon$ is the extinction coefficient of the fluorescence agent at the excitation wavelength.

\section{A. Pharmacokinetic Rate to Measurement Map for Fluores- cence Agents}

To derive a complete formulation to reconstruct the pharmacokinetic parameters and concentrations in different compartments using the photon flux measurements, we combined the FDOT forward problem equations, (10) or (11) with the compartmental model equations.

Combining (9) and (10), with the knowledge of the linear relationship between the absorption coefficient and the bulk fluorescence concentration, (12), nonlinear FDOT forward problem is related to the bulk fluorescence agent concentration as:

$$
\boldsymbol{\Psi}(\mathbf{r}, t)=g(\mathbf{m}(\mathbf{r}, t)) \sim g\left(\mathbf{V}\left(\boldsymbol{\alpha}_{n}(\mathbf{r})\right) \mathbf{C}(\mathbf{r}, t)\right)
$$

where $g$ is the non-linear function which relates the bulk fluorescence agent concentrations to the boundary flux measurements.

The linearized version of (11) is given as:

$$
\begin{aligned}
\Psi(\mathbf{r}, t) & =\epsilon \mathbf{W m}(\mathbf{r}, t) \\
& =\epsilon \mathbf{W V}\left(\boldsymbol{\alpha}_{n}(\mathbf{r})\right) \mathbf{C}(\mathbf{r}, t)+\epsilon \mathbf{W} \boldsymbol{\eta}(\mathbf{r}, t) \\
& =\Gamma \mathbf{C}(\mathbf{r}, t)+\epsilon \mathbf{W} \boldsymbol{\eta}(\mathbf{r}, t),
\end{aligned}
$$

where $\mathbf{W}, \mathbf{V}$ and $\boldsymbol{\eta}(\mathbf{r}, t)$ are defined as above and $\Gamma=$ $\epsilon \mathbf{W V}\left(\boldsymbol{\alpha}_{n}(\mathbf{r})\right)$.

The equations (8) and (13), which combine the FDOT forward problem with the compartment model equations constitute the set of equations which will be used for the direct reconstruction of pharmacokinetic rates, volume fractions, and fluorescence concentrations in different compartments.

\section{Direct Reconstruction of Pharmacokinetic RATE AND CONCENTRATION IMAGES FROM OPTICAL Flux MEASUREMENTS}

The source detector measurements in (9) are collected at discrete time instances, $t=k T, k=0,1, \ldots$, where $T$ is the sampling period. Therefore, the continuous model described in (8) and (14) has to be discretized. To simplify our notation, we shall use $\mathbf{C}(\mathbf{r}, k)=\mathbf{C}(\mathbf{r}, k T)$ and $\boldsymbol{\Psi}(\mathbf{r}, k)=\boldsymbol{\Psi}(\mathbf{r}, k T)$.

Let $\boldsymbol{\theta}_{n}$ denote the discrete-time parameter vector of the pharmacokinetic rates and volume fractions. The parameter vector $\boldsymbol{\theta}_{n}(\mathbf{r})$ can be either time dependent or time independent. The formulation given in this work can be used for both cases. In our case, the pharmacokinetic rates and the volume fractions are time independent. However, in order to estimate $\boldsymbol{\theta}_{n}$ within the EKF framework, the following dynamic model is introduced:

$$
\boldsymbol{\theta}_{n}(\mathbf{r}, k+1)=\boldsymbol{\theta}_{n}(\mathbf{r}, k)+\boldsymbol{\varsigma}(\mathbf{r}, k),
$$

where $\boldsymbol{\varsigma}(\mathbf{r}, k)$ is a zero mean white noise process with covariance matrix $\mathbf{S}$. The details of the dynamic model introduced for the joint estimation of the system parameters and the states can be found in [3].
1) A priori information for Pharmacokinetic Rates and Volume Fractions: To improve the robustness of estimates of the parameters, we impose a priori information on pharmacokinetic parameters and volume fractions. Here, we assume that there is no information about the tumor structure and used a 4-pixel neighborhood model.

Using the 4-pixel neighbor model with equal weights $\beta$, the random process, $\boldsymbol{\theta}_{n}(\mathbf{r}, k)$, can be modeled as:

$$
\boldsymbol{\theta}_{n}\left(r_{j}, k+1\right)=\beta \boldsymbol{\theta}_{n}\left(r_{j}, k\right)+\beta \sum_{i=1}^{4} \boldsymbol{\theta}_{n}\left(r_{j i}, k\right)+\boldsymbol{\varsigma}(\mathbf{r}, k) .
$$

2) Extended Kalman Filter (EKF) Formulation: In our state-space model, (8) and (9), both the states (concentrations) and system parameters (pharmacokinetic rates and volume fractions) are unknown. In this case, the state-space model can be regarded as a non-linear model in which system parameters and states are combined to form the new states of the nonlinear model. This system is then linearized and solved for the unknown states using EKF framework [3]. To solve for the concentrations and the unknown parameters, the parameter vector $\boldsymbol{\theta}_{n}(\mathbf{r}, k)$ is inserted into the concentration vector $\mathbf{C}(\mathbf{r}, k)$ as:

$\left[\begin{array}{c}\mathbf{C}(\mathbf{r}, k+1) \\ \boldsymbol{\theta}_{n}\left(r_{j}, k+1\right)\end{array}\right]=\left[\begin{array}{c}\mathbf{K}\left(\boldsymbol{\theta}_{n}(\mathbf{r}, k)\right) \mathbf{C}(\mathbf{r}, k) \\ \beta \boldsymbol{\theta}_{n}\left(r_{j}, k\right)+\beta \sum_{i=1}^{4} \boldsymbol{\theta}_{n}\left(r_{j i}, k\right)\end{array}\right]+\left[\begin{array}{c}\boldsymbol{\omega}(\mathbf{r}, k) \\ \boldsymbol{\varsigma}(\mathbf{r}, k)\end{array}\right]$.

The measurement equation for the non-linear case is given as:

$\boldsymbol{\Psi}(\mathbf{r}, k)=\left[g\left(\mathbf{V}\left(\boldsymbol{\alpha}_{n}(\mathbf{r})\right) \mathbf{C}(\mathbf{r}, k)\right) \quad 0\right]\left[\begin{array}{c}\mathbf{C}(\mathbf{r}, k) \\ \boldsymbol{\theta}_{n}(\mathbf{r}, k)\end{array}\right]+\eta(\mathbf{r}, k)$.

Without explicit proof, the extended Kalman filtering algorithm for simultaneous estimation of concentrations and parameters for the nonlinear case, (13), is given follows:

$$
\begin{aligned}
& {\left[\begin{array}{c}
\hat{\mathbf{C}}(\mathbf{r}, 0) \\
\hat{\boldsymbol{\theta}}_{n}(\mathbf{r}, 0)
\end{array}\right]=\left[\begin{array}{c}
E(\mathbf{C}(\mathbf{r}, 0)) \\
\hat{\boldsymbol{\theta}}_{n}(\mathbf{r}, 0)
\end{array}\right] .} \\
& \mathbf{P}_{0,0}=\left[\begin{array}{cc}
\operatorname{Var}(\mathbf{C}(\mathbf{r}, 0)) & 0 \\
0 & \mathbf{S}_{d}
\end{array}\right],
\end{aligned}
$$

where $E$ denotes the expected value of $\mathbf{C}(\mathbf{r}, 0), \mathbf{P}$ is the error covariance matrix, and $\mathbf{S}_{d}$ is the preassigned covariance matrix of the unknown system parameters.

The following equations describe how the concentration estimates and error covariance matrix are updated at the $k^{t h}$ time instant given all the measurements up to $(k-1)^{t h}$ time instant. For $k=1,2, \ldots$,

$$
\left[\begin{array}{c}
\hat{\mathbf{C}}(\mathbf{r}, k \mid k-1) \\
\hat{\boldsymbol{\theta}}_{n}\left(r_{j}, k \mid k-1\right)
\end{array}\right]=\left[\begin{array}{c}
\mathbf{K}\left(\hat{\boldsymbol{\theta}}_{n}(r, k-1) \hat{\mathbf{C}}(\mathbf{r}, k-1)\right. \\
\beta \hat{\boldsymbol{\theta}}_{n}\left(r_{j}, k-1\right)+\beta \sum_{i=1}^{4} \hat{\boldsymbol{\theta}}_{n}\left(r_{j i}, k\right)
\end{array}\right]
$$$$
\mathbf{P}_{k \mid k-1}=\mathbf{J}(k-1) \mathbf{P}_{k-1, k-1} \mathbf{J}^{T}(k-1)+\left[\begin{array}{cc}
\mathbf{Q}_{d} & 0 \\
0 & \mathbf{S}_{d}
\end{array}\right],
$$

where $\mathbf{J}$ is the Jacobian matrix given by

$$
\mathbf{J}(k)=\left[\begin{array}{cc}
\mathbf{K}\left(\hat{\boldsymbol{\theta}}_{n}(\mathbf{r}, k)\right) & \frac{\partial}{\partial \boldsymbol{\theta}}\left[\mathbf{K}\left(\hat{\boldsymbol{\theta}}_{n}(\mathbf{r}, k)\right) \hat{\mathbf{C}}(\mathbf{r}, k)\right] \\
\frac{\partial}{\partial \mathbf{C}} \hat{\boldsymbol{\theta}}_{n}(\mathbf{r}, k) & \frac{\partial}{\partial \hat{\boldsymbol{\theta}}_{n}} \hat{\boldsymbol{\theta}}_{n}(\mathbf{r}, k)
\end{array}\right] .
$$




$$
\mathbf{G}_{k}=\mathbf{P}_{k, k-1} \boldsymbol{\Lambda}^{T}\left[\boldsymbol{\Lambda} \mathbf{P}_{k, k-1} \boldsymbol{\Lambda}^{T}+\mathbf{R}\right]^{-1},
$$

where $\mathbf{G}_{k}$ is the recursive Kalman gain, $\mathbf{R}$ is the covariance matrix of the measurements and $\Lambda$ is:

$$
\boldsymbol{\Lambda}=\left[\begin{array}{c}
\frac{\partial}{\partial \mathbf{C}} g\left(\mathbf{V}\left(\boldsymbol{\theta}_{n}(\mathbf{r}, k \mid k-1) \mathbf{C}(\mathbf{r}, k \mid k-1)\right)\right. \\
\cdot \cdot \frac{\partial}{\partial \boldsymbol{\theta}}\left(\frac { \partial } { \partial \mathbf { C } } g \left(\mathbf { V } \left(\boldsymbol{\theta}_{n}(\mathbf{r}, k \mid k-1) \mathbf{C}(\mathbf{r}, k \mid k-1)\right.\right.\right.
\end{array}\right]^{T},
$$

where $\mathbf{I}$ is the identity matrix.

$$
\begin{array}{r}
{\left[\begin{array}{c}
\hat{\mathbf{C}}(\mathbf{r}, k) \\
\hat{\boldsymbol{\theta}}_{n}\left(\mathbf{r}_{j}, k\right)
\end{array}\right]=\left[\begin{array}{c}
\hat{\mathbf{C}}(\mathbf{r}, k \mid k-1) \\
\beta \hat{\boldsymbol{\theta}}_{n}\left(r_{j}, k \mid k-1\right)+\beta \sum_{i=1}^{4} \hat{\boldsymbol{\theta}}_{n}\left(r_{j i}, k \mid k-1\right)
\end{array}\right]} \\
+\mathbf{G}_{k}\left(\mathbf{\Psi}(\mathbf{r}, k)-g\left(\mathbf{V}\left(\boldsymbol{\theta}_{n}(\mathbf{r}, k \mid k-1) \mathbf{C}(\mathbf{r}, k \mid k-1)\right) .\right.\right.
\end{array}
$$

\section{Simulations And Results}

To validate our approach, we performed a simulation study based on the two-compartment model proposed for ICG. Using physiologically correct values for pharmacokinetic rates, $k_{i n}$, $k_{\text {out }}, k_{\text {elm }}$, and volume fractions, $v_{e}, v_{p}$, around the tumor region, a set of time series data, $\boldsymbol{\Psi}(\mathbf{r}, t)$, was generated from a simulated domain with tissue-like characteristics. To generate the synthetic measurements, the diffusion equation was solved numerically using FEM algorithm with Robin type boundary conditions [10]. The simulation used a modulation frequency $300 \mathrm{MHz}$. The phantom is $6 \mathrm{~cm}$ by $6 \mathrm{~cm}$ in size, and it is discretized into 24 by 24 voxels each of size $0.25 \mathrm{~cm}$ by $0.25 \mathrm{~cm}$. The 24 sources and 24 detectors are arranged
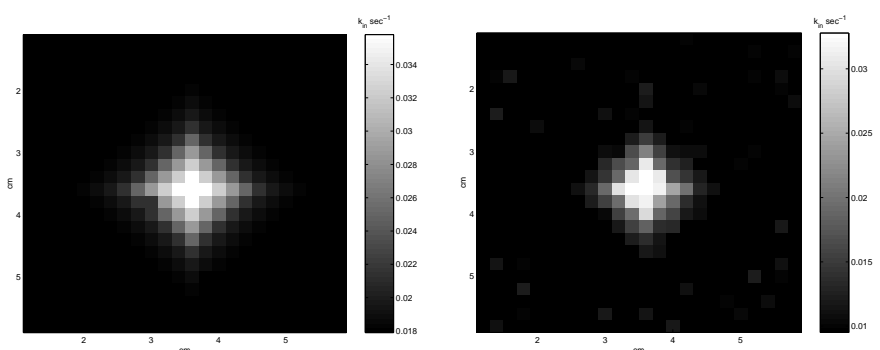

Fig. 2. Pharmacokinetic rate images of $k_{i n}$ : (a) true, and (b) reconstructed.
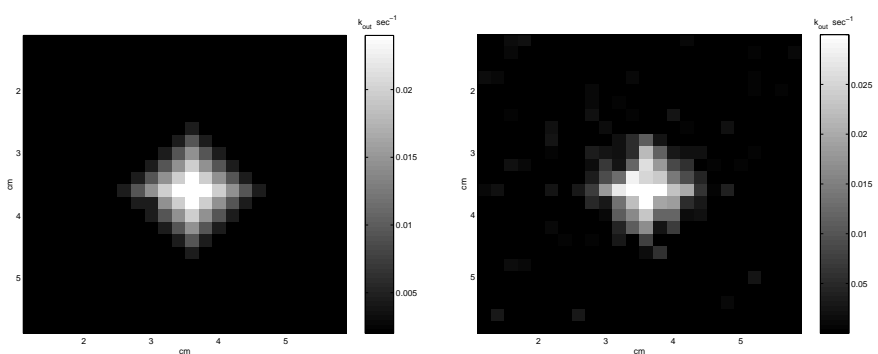

Fig. 3. Pharmacokinetic rate images of $k_{o u t}$ : (a) true, and (b) reconstructed.

throughout the boundary sequentially. The maximum transition rates of $k_{i n}$ and $k_{\text {out }}$ are simulated at the center of the image of size $1 \mathrm{~cm}$ by $1 \mathrm{~cm}$ and smoothly decreased towards the boundaries. Figures $2 \mathrm{a}$ and $3 \mathrm{a}$ display the true images of pharmacokinetic rates $k_{i n}$ and $k_{\text {out }}$. Figures $2 \mathrm{~b}$ and $3 \mathrm{~b}$ display the corresponding reconstructed images. We observe that there is a good agreement between the true and the estimated images in terms of localization of the heterogeneities. We calculated the percent error between the true images and the reconstructed images by the ratio of the $L^{2}$ norm of the error and the $L^{2}$ norm of the true image. The percent error for $k_{i n}$ and $k_{\text {out }}$ are $11.95 \%$ and $13.11 \%$, respectively.

\section{CONCLUSiON}

In this paper, we provided a formulation for the reconstruction of spatially resolved pharmacokinetic rate images of fluorescence agents directly from the boundary photon flux measurements. We performed a simulation study using a numerical phantom. Reconstructed images with small errors show that the algorithm can be used for real data analysis. In the near feature, we plan to apply the proposed algorithm to the ICG concentration data acquired from breast tumors.

\section{REFERENCES}

[1] E. M. Sevick-Muraca et al., "Fluorescence and absorption contrast mechanisms for biomedical optical imaging using frequency-domain techniques," Photochem. Photobiol., Vol.66, pp. 55, 1997.

[2] L. A. Bauer, Applied Clinical Pharmacokinetics', McGraw-Hill, New York, 2001

[3] B. Alacam, B. Yazici, X. Intes, B. Chance, ” Extended Kalman Filtering for the Modeling and Analysis of ICG Pharmacokinetics in Cancerous Tumors using NIR Optical Methods", IEEE Tran. in Biomedical Engineering, in Press.

[4] D.J. Cuccia et al., "In vivo quantification of optical contrast agent dynamics in rat tumors by use of diffuse optical spectroscopy with magnetic resonance imaging coregistration," Applied Optics, vol. 42, no. 1, pp. 2940-2950, June 2003.

[5] X. Intes, J. Ripoll, Y. Chen, S. Nioka, A. G. Yodh, B. Chance, "In vivo continuous-wave optical breast imaging enhanced with Indocyanine Green," Med. Phys., vol. 30-6, pp.1039-1047, June 2003.

[6] C. Cobelli, D. Foster, and G. Toffolo, Tracer Kinetics in Biomedical Research, Kluwer Academic/Plenum, New York, c2000.

[7] M. Kamasak et al., "Direct reconstruction of kinetic parameter images from dynamic PET data," $37^{\text {th }}$ Asilomar Conf. on Signals, Systems and Computers, Pacific Grove, CA, pp. 19191923, 2003.

[8] A. B. Milstein, K. J. Webb, and C. A. Bouman, "Estimation of kinetic model parameters in fluorescence optical diffusion tomography," J. Opt. Soc. Am., Vol. 22, No. 7, pp. 1357-1368.

[9] B. Alacam et al., "Spatially Resolved Pharmacokinetic Rate Images of ICG using Near Infrared Optical Methods", Proc. of 2006 SPIE Photonic West, San Jose, CA, 21-26 January 2006, In Press.

[10] S. C. Brenner, and L. R. Scott, The mathematical theory of finite element methods, Springer, Berlin, 1994. 